\title{
Analytical approximations for the dispersion of electromagnetic modes in slabs of biaxial crystals
}

\author{
Gonzalo Álvarez-Pérez $\bullet,{ }^{1,2}$ Kirill V. Voronin $\odot,{ }^{3}$ Valentyn S. Volkov, ${ }^{3}$ \\ Pablo Alonso-González $\odot,{ }^{1,2, *}$ and Alexey Y. Nikitin ${ }^{4,5,3, \dagger}$ \\ ${ }^{1}$ Department of Physics, University of Oviedo, Oviedo 33006, Spain \\ ${ }^{2}$ Center of Research on Nanomaterials and Nanotechnology, CINN (CSIC-Universidad de Oviedo), El Entrego 33940, Spain \\ ${ }^{3}$ Center for Photonics and 2D Materials, Moscow Institute of Physics and Technology, Dolgoprudny 141700, Russia \\ ${ }^{4}$ Donostia International Physics Center (DIPC), Donostia-San Sebastán 20018, Spain \\ ${ }^{5}$ IKERBASQUE, Basque Foundation for Science, Bilbao 48013, Spain
}

(Received 13 September 2019; revised manuscript received 13 November 2019; published 5 December 2019)

\begin{abstract}
Anisotropic crystals have recently attracted considerable attention because of their ability to support polaritons with a variety of unique properties, such as hyperbolic dispersion, negative phase velocity, or extreme confinement. Particularly, the biaxial crystal $\alpha-\mathrm{MoO}_{3}$ has been demonstrated to support phonon polaritons, light coupled to lattice vibrations, with in-plane anisotropic propagation and unusually long lifetime. However, the lack of theoretical studies on electromagnetic modes in biaxial crystal slabs impedes a complete interpretation of the experimental data, as well as an efficient design of nanostructures supporting such highly anisotropic polaritons. Here, we derive the dispersion relation of electromagnetic modes in biaxial slabs surrounded by semi-infinite isotropic dielectric half-spaces with arbitrary dielectric permittivities. Apart from a general dispersion relation, we provide very simple analytical expressions in typical experiments in nano-optics: the limits of short polaritonic wavelength and/or very thin slabs. The results of our study will allow for an in-depth analysis of anisotropic polaritons in novel biaxial van der Waals materials.
\end{abstract}

DOI: 10.1103/PhysRevB.100.235408

\section{INTRODUCTION}

Anisotropic media have been a subject of fundamental and applied research in optics for several centuries since the earliest Bartholinus' studies. Particularly, birefringence, responsible for the double refraction inside anisotropic crystals, is widely used nowadays in daily-life applications requiring polarization filtering as, for instance, sun glasses, liquidcrystal displays, or scanning laser polarimetry (for monitoring glaucoma) [1]. In recent decades, the scientific interest to anisotropic optical phenomena has dramatically increased due to the design and fabrication of novel artificial materials (metamaterials) with a tailored optical response. Striking examples of the latter are photonic and plasmonic crystals [2-4] and metasurfaces [5,6], showing spectacular phenomena such as negative refraction [7], slow light [8], and superlensing [9,10], among others. Apart from these anisotropic artificial materials, a few years ago the concept of "atomic-scale" engineering with naturally anisotropic van der Waals ( $\mathrm{vdW}$ ) materials [11] was suggested, adding more scientific interest to this field. Currently, presenting one of the main strategies in low-dimensional optoelectronics, this concept has induced an intensive study of highly confined anisotropic polaritons supported by vdW slabs and heterostructures $[12,13]$. The possibility of visualizing these polaritons in thin slabs of vdW crystals with the use of near-field microscopy [14-18]

\footnotetext{
*Corresponding author: pabloalonso@uniovi.es
}

$\dagger$ Corresponding author: alexey@ dipc.org stimulates more and more experimental and theoretical studies in this direction.

From a theoretical point of view, in bulk uniaxial crystals (such as $h-\mathrm{BN}, \mathrm{SiC}$, or layered metamaterials), characterized by two refractive indices, electromagnetic eigenmodes present ordinary and extraordinary waves. In many cases, the propagation of light along the boundaries of uniaxial crystals and inside the slabs can be straightforwardly analyzed analytically $[14,19,20]$. In stark contrast, biaxial crystals (such as $\alpha-\mathrm{MoO}_{3}$ or $\mathrm{V}_{2} \mathrm{O}_{5}$ ) are characterized by three refractive indices and both electromagnetic eigenmodes are extraordinary. As a result, the understanding and analytical treatment of electromagnetic phenomena in biaxial media is significantly more complex than in the uniaxial case. In this context, a very recent study has reported on a rigorous analytical solution for the dispersion of surface waves on the boundaries of biaxial crystals [21]; however, up to now, studies on the electromagnetic modes in biaxial slabs have been mainly the subject of a numerical analysis [22-24] or some particular configurations, such as grounded crystal slabs with mode fixed propagation directions [25].

In this work, organized in a tutorial style, we present a detailed derivation of the dispersion relation of electromagnetic modes in a biaxial slab of a finite thickness (with arbitrary dielectric tensor), surrounded by two semi-infinite isotropic media with arbitrary dielectric permittivities. We assume that one of the principal crystal axes is perpendicular to the faces of the slab, while the mode propagates along the crystal slab at an arbitrary angle with respect to the other principal axes, which lays in a plane parallel to the faces of the slab. We show that our general dispersion relation successfully reduces 
(a)

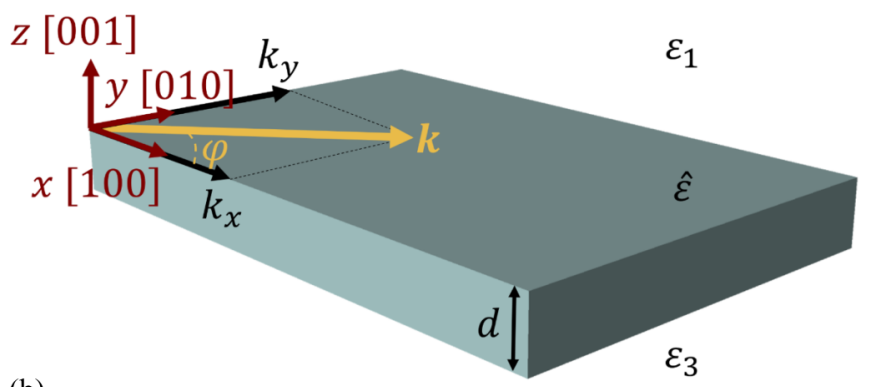

(b)

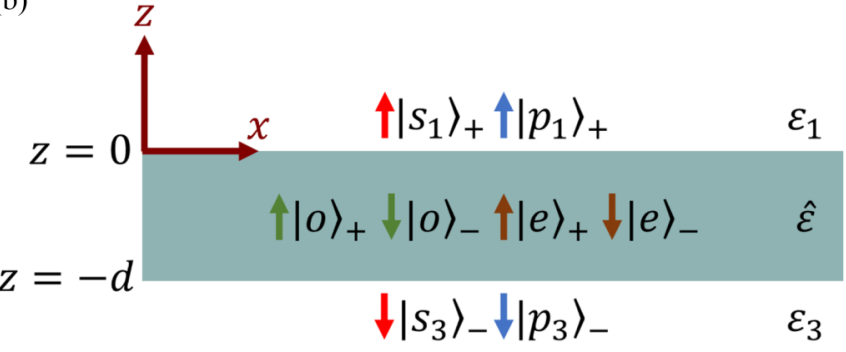

FIG. 1. Schematics of the biaxial slab. One of the main crystal axes $[0,0,1]$ is perpendicular to the faces of the slab and coincides with the $z$ axis, while the axes $[1,0,0]$ and $[0,1,0]$ belong to a plane parallel to the faces of the slab and are directed along the coordinate axes $x$ and $y$, respectively. The mode propagates at an arbitrary angle $\varphi$ with respect to the $x$ axis.

to the known limiting cases, such as the case of a uniaxial slab or a semi-infinite crystal, among others. We manage to reduce the general dispersion relation to simple analytical expressions for short wavelength of the modes and small slab thicknesses, which are currently of great interest for the study of anisotropic polaritons in vdW slabs. To demonstrate the validity of our analytical approximations, we compare them to full-wave simulations, finding an excellent agreement.

\section{INFINITE BIAXIAL CRYSTAL}

Let us consider an infinite, nonmagnetic biaxial medium with dielectric permittivity tensor $\hat{\varepsilon}$. The coordinate system $\{x, y, z\}$ is chosen is such a way that $\hat{\varepsilon}$ is diagonal [see Fig. 1(a)], so that

$$
\hat{\varepsilon}=\left(\begin{array}{ccc}
\varepsilon_{x} & 0 & 0 \\
0 & \varepsilon_{y} & 0 \\
0 & 0 & \varepsilon_{z}
\end{array}\right)
$$

To accurately decompose the electromagnetic fields in the biaxial medium, we need to define appropriate basis vectors. To that end, we follow a standard procedure, as for example in Ref. [21]. Namely, we represent the electric and magnetic fields in the biaxial medium in the form of plane waves:

$$
\mathbf{E}=E_{0} \mathbf{e} e^{i \mathbf{k r}-i \omega t}, \quad \mathbf{H}=H_{0} \mathbf{h} e^{i \mathbf{k r}-i \omega t},
$$

where $\mathbf{e}$ and $\mathbf{h}$ are unknown dimensionless field basis vectors, $E_{0}$ and $H_{0}$ are arbitrary field amplitude coefficients, $\omega$ is the angular frequency, $\mathbf{k}$ is the wave vector, and $\mathbf{r}$ is the radius vector.

From Maxwell's equations $\left(\boldsymbol{\nabla} \times \mathbf{E}=-\frac{1}{c} \frac{\partial \mathbf{H}}{\partial t}\right.$ and $\boldsymbol{\nabla} \times$ $\mathbf{H}=\frac{1}{c} \frac{\partial \mathbf{D}}{\partial t}$ ), and substituting the magnetic field $\mathbf{H}$, we obtain a vectorial equation for the electric fields $\mathbf{E}$ :

$$
\frac{\omega^{2}}{c^{2}} \hat{\varepsilon} \mathbf{E}=\nabla(\nabla \mathbf{E})-\Delta \mathbf{E} .
$$

Substituting E from Eq. (2) into Eq. (3), we obtain a linear homogeneous system of equations for the three components of the unknown basis vector $\mathbf{e}$ :

$$
\mathcal{M} \mathbf{e}=\left(\begin{array}{ccc}
\Delta_{x} & q_{x} q_{y} & \pm i q_{x} q_{z} \\
q_{x} q_{y} & \Delta_{y} & \pm i q_{y} q_{z} \\
\pm i q_{x} q_{z} & \pm i q_{y} q_{z} & \Delta_{z}
\end{array}\right)\left(\begin{array}{l}
e_{x} \\
e_{y} \\
e_{z}
\end{array}\right)=0
$$

where $k_{0}=\omega / c$ is the free-space wave vector, $q_{x, y}=k_{x, y} / k_{0}$ are the in-plane components of the normalized wave vector, and $q_{z}$ is the out-of-plane component of the normalized wave vector, so that $k_{z}= \pm i q_{z} k_{0}$. The $+(-)$ sign must be taken for the wave propagating along (opposite to) the $z$ axis, while $\Delta_{i}$ are defined as

$$
\begin{aligned}
& \Delta_{x}=\varepsilon_{x}-q_{y}^{2}+q_{z}^{2}, \\
& \Delta_{y}=\varepsilon_{y}-q_{x}^{2}+q_{z}^{2}, \\
& \Delta_{z}=\varepsilon_{z}-q_{x}^{2}-q_{y}^{2} .
\end{aligned}
$$

Our choice of the dependence of the basis vectors upon the coordinates (the propagation along $z$ is treated differently from the propagation along $x$ and $y$ ) is dictated by the geometry of the problem (see Fig. 1), consistently with the standard waveguide theory.

The system (4) has nontrivial solutions only when $\operatorname{det}(\mathcal{M})=0$, that gives the well-known Fresnel's equation for biaxial media $[26,27]$

$$
\begin{gathered}
q_{z}^{2}\left[q_{z}^{2} \varepsilon_{z}+\varepsilon_{z}\left(\varepsilon_{x}+\varepsilon_{y}\right)-q_{x}^{2}\left(\varepsilon_{x}+\varepsilon_{z}\right)-q_{y}^{2}\left(\varepsilon_{y}+\varepsilon_{z}\right)\right] \\
\quad+\left(\varepsilon_{z}-q_{x}^{2}-q_{y}^{2}\right)\left(\varepsilon_{x} \varepsilon_{y}-q_{x}^{2} \varepsilon_{x}-q_{y}^{2} \varepsilon_{y}\right)=0 .
\end{gathered}
$$

It is the quadratic equation in terms of the squared $z$ component of the wave vector, $q_{z}$. Its solutions $q_{e z}$ and $q_{o z}$ read as

$$
q_{o, e z}^{2}=\frac{1}{2}\left\{\frac{\varepsilon_{x}+\varepsilon_{z}}{\varepsilon_{z}} q_{x}^{2}+\frac{\varepsilon_{y}+\varepsilon_{z}}{\varepsilon_{z}} q_{y}^{2}-\left(\varepsilon_{x}+\varepsilon_{y}\right)\right\} \pm \frac{1}{2} \sqrt{D},
$$

with $D$ being the discriminant

$$
\begin{aligned}
D= & \left(\varepsilon_{x}-\varepsilon_{y}+\frac{\varepsilon_{z}-\varepsilon_{x}}{\varepsilon_{z}} q_{x}^{2}-\frac{\varepsilon_{z}-\varepsilon_{y}}{\varepsilon_{z}} q_{y}^{2}\right)^{2} \\
& +4 \frac{\left(\varepsilon_{z}-\varepsilon_{x}\right)\left(\varepsilon_{z}-\varepsilon_{y}\right)}{\varepsilon_{z}^{2}} q_{x}^{2} q_{y}^{2} .
\end{aligned}
$$

In Eq. (7), the signs "+" and "-" correspond to the labels " $o$ " and " $e$ ", respectively. Substituting Eq. (7) into the system (4), we find all three components of the two eigenvectors e. Since the system (4) is homogeneous, one of the components of the eigenvectors must be fixed. Without loss of generality, fixing the $y$ component to $e_{y}=q_{x}$ for the root $o$ and to $e_{y}=q_{y}$ for the root $e$, we find

$$
\mathbf{e}_{o}=\frac{1}{q}\left(\begin{array}{c}
-q_{y}\left(1-\Delta_{1} \Delta_{z}\right) \\
q_{x} \\
\mp i q_{x} q_{y} q_{o z} \Delta_{1}
\end{array}\right), \quad \mathbf{e}_{e}=\frac{1}{q}\left(\begin{array}{c}
q_{x} \frac{\Delta_{2}-q_{y}^{2}}{\Delta_{x}^{e}} \\
q_{y} \\
\frac{\Delta_{2}}{\mp i q_{e z}}
\end{array}\right),
$$


where the factor $1 / q$ ( $q$ being the normalized in-plane wave vector $q^{2}=\frac{k_{x}^{2}+k_{y}^{2}}{k_{0}^{2}}$ ) stands for the normalization, and

$$
\Delta_{1}=\frac{\Delta_{x}^{o}-q_{x}^{2}}{\Delta_{z} \Delta_{x}^{o}+q_{x}^{2} q_{o z}^{2}}, \quad \Delta_{2}=\frac{\Delta_{x}^{e} \Delta_{y}^{e}-q_{x}^{2} q_{y}^{2}}{\Delta_{x}^{e}-q_{x}^{2}} .
$$

From Eq. (6) we can easily find the asymptotes of the isofrequency curves for large $q_{x, y}$. Tending both $q_{x}$ and $q_{y}$ to infinity, and setting $q_{z}=0$, we find

$$
\frac{q_{x}}{q_{y}}=\sqrt{-\frac{\varepsilon_{y}}{\varepsilon_{x}}} .
$$

In thin vdW slabs these asymptotes yield the direction of the propagation of the polaritonic "rays," excited by localized sources [15-17]. In the particular case of a uniaxial crystal (with the axis $C$ pointing parallel to the $z$ axis, $C \| O z$ ), $\varepsilon_{x}=\varepsilon_{y}=\varepsilon_{\perp}$ and $\varepsilon_{z}=\varepsilon_{\|}$, the derived basis vectors (9) can be straightforwardly transformed to the basis vectors for the ordinary and extraordinary waves. Taking into account that the $z$ components of the wave vectors (7) are reduced to the wellknown expressions for the ordinary and extraordinary waves

$$
q_{o z}^{2}=q^{2}-\varepsilon_{\perp}, \quad q_{e z}^{2}=\frac{\varepsilon_{\perp}}{\varepsilon_{\|}} q^{2}-\varepsilon_{\perp},
$$

we find that $\Delta_{1}=0, \Delta_{2}=\varepsilon_{\perp}+q_{e z}^{2}$ and obtain the basis vectors

$$
\mathbf{e}_{o}=\frac{1}{q}\left(\begin{array}{c}
-q_{y} \\
q_{x} \\
0
\end{array}\right), \quad \mathbf{e}_{e}=\frac{1}{q}\left(\begin{array}{c}
q_{x} \\
q_{y} \\
\frac{\varepsilon_{\perp}+q_{e z}^{2}}{\mp i q_{e z}}
\end{array}\right) .
$$

In case of an isotropic medium, $\varepsilon_{\perp}=\varepsilon_{\|}=\varepsilon$, the $z$ components of the wave vectors degenerate $q_{o z}^{2}=q_{e z}^{2}=$ $q_{z}^{2}=q^{2}-\varepsilon$ and the basis vectors (9) reduce to the ones for the $s$ - and $p$-polarized waves $\left(\mathbf{e}_{o} \rightarrow \mathbf{e}_{s}\right.$ and $\mathbf{e}_{e} \rightarrow \mathbf{e}_{p}$ ):

$$
\mathbf{e}_{s}=\frac{1}{q}\left(\begin{array}{c}
-q_{y} \\
q_{x} \\
0
\end{array}\right), \quad \mathbf{e}_{p}=\frac{1}{q}\left(\begin{array}{c}
q_{x} \\
q_{y} \\
\frac{q^{2}}{\mp i q_{z}}
\end{array}\right) .
$$

\section{BIAXIAL SLAB OF A FINITE THICKNESS}

Here, we derive the dispersion relation for polaritons in a biaxial slab of thickness $d$ and permittivity $\hat{\varepsilon}$, occupying the region $0>z>-d$ between two dielectric half-spaces with permittivities $\varepsilon_{1}$ (region " $1, " z>0$ ) and $\varepsilon_{3}$ (region "3," $z<-d)$.

\section{A. General form of the dispersion relation}

Let us first represent the electric fields above $(z>0)$ and below $(z<-d)$ the slab, in the isotropic media "1" and "3," respectively. In these regions we can take the fields in the form of the $s$ - and $p$-polarized plane waves. For compactness, from now on, we will use Dirac notation, in which the $s$ - and $p$ polarization basis vectors read as

$\left|s_{1,3}\right\rangle_{ \pm}=\frac{1}{q}\left(\begin{array}{c}-q_{y} \\ q_{x} \\ 0\end{array}\right) e^{i k_{x} x+i k_{y} y}, \quad\left|p_{1,3}\right\rangle_{ \pm}=\frac{1}{q}\left(\begin{array}{c}q_{x} \\ q_{y} \\ \frac{q^{2}}{\mp i q_{1,3 z}}\end{array}\right) e^{i k_{x} x+i k_{y} y}$, where $q_{1,3 z}=\sqrt{q_{x}^{2}+q_{y}^{2}-\varepsilon_{1,3}}>0$ is the out-of-plane component of the normalized wave vector. In the definition of the $p$-polarization basis vector, $\left|p_{1,3}\right\rangle_{ \pm}$, the $+(-)$sign should be taken for the wave propagating along (opposite to) the $z$ axis, while in case of the $s$ polarization, $\left|s_{1,3}\right\rangle_{+}$and $\left|s_{1,3}\right\rangle_{-}$are degenerated. For convenience, we also introduce the in-plane subvectors of the vectors given by Eq. (15):

$$
|s\rangle=\frac{1}{q}\left(\begin{array}{c}
-q_{y} \\
q_{x}
\end{array}\right) e^{i k_{x} x+i k_{y} y}, \quad|p\rangle=\frac{1}{q}\left(\begin{array}{c}
q_{x} \\
q_{y}
\end{array}\right) e^{i k_{x} x+i k_{y} y} .
$$

The fields of the mode propagating along the slab in the upper and lower media can be compactly written as the sum of the $s$ - and $p$-polarized plane waves:

$$
\begin{aligned}
& \mathbf{E}_{1}=\mathbf{E}_{1}(x, y, z)=\sum_{\beta=s, p} a_{\beta}^{1}\left|\beta_{1}\right\rangle_{+} e^{i k_{z} z}, \\
& \mathbf{E}_{3}=\mathbf{E}_{3}(x, y, z)=\sum_{\beta=s, p} a_{\beta}^{3}\left|\beta_{3}\right\rangle_{-} e^{-i k_{z} z},
\end{aligned}
$$

with unknown amplitudes $a_{\beta}^{1,3}$.

In contrast, the electric fields inside the biaxial slab $(0>$ $z>-d$ ) should be represented with the help of the basis vectors found in Sec. II as

$$
\mathbf{E}_{2}=\mathbf{E}_{2}(x, y, z)=\sum_{\gamma=o, e} a_{\gamma}^{2 \downarrow}|\gamma\rangle_{+} e^{i k_{\gamma} z}+a_{\gamma}^{2 \uparrow}|\gamma\rangle_{-} e^{-i k_{\gamma z} z},
$$

where $|\gamma\rangle_{ \pm}$denotes $|o\rangle_{ \pm}$and $|e\rangle_{ \pm}$, being the polarization basis vector in the biaxial slab

$$
\begin{aligned}
& |o\rangle_{ \pm}=\frac{1}{q}\left(\begin{array}{c}
-q_{y}\left(1-\Delta_{1} \Delta_{z}\right) \\
q_{x} \\
\mp i q_{x} q_{y} q_{o z} \Delta_{1}
\end{array}\right) e^{i k_{x} x+i k_{y} y}, \\
& |e\rangle_{ \pm}=\frac{1}{q}\left(\begin{array}{c}
q_{x} \frac{\Delta_{2}-q_{y}^{2}}{\Delta_{x}^{e}} \\
q_{y}^{\Delta_{2}} \\
\mp i q_{e z}
\end{array}\right) e^{i k_{x} x+i k_{y} y} .
\end{aligned}
$$

The factors $a_{\gamma}^{2 \uparrow}$ and $a_{\gamma}^{2 \downarrow}$ represent the unknown amplitudes of the plane waves traveling along and opposite to the $z$ axis, respectively. Analogously to isotropic regions, we can introduce the in-plane subvectors $|o\rangle$ and $|e\rangle$ of the vectors $|o\rangle_{ \pm},|e\rangle_{ \pm}$, respectively. These subvectors can be compactly written as

$$
|o\rangle=|s\rangle+\frac{q_{y} c_{1}}{q}\left|u_{x}\right\rangle, \quad|e\rangle=|p\rangle+\frac{q_{x} c_{2}}{q}\left|u_{x}\right\rangle,
$$

where $\left|u_{x}\right\rangle=(1,0)^{T}$, and

$$
c_{1}=\Delta_{1} \Delta_{z}, \quad c_{2}=\left(\frac{\Delta_{2}-q_{y}^{2}}{\Delta_{x}^{e}}-1\right) .
$$

To find the magnetic fields, we use Maxwell's equation $\nabla \times \mathbf{E}=-\frac{1}{c} \frac{\partial \mathbf{H}}{\partial t}$. In case of a plane wave it simplifies to

$$
\mathbf{H}=\mathbf{q}_{i} \times \mathbf{E},
$$

where $\mathbf{q}_{i}$ is the normalized wave vector of a corresponding plane wave. Then, we can apply the boundary conditions, which imply the continuity of the in-plane components of both electric and magnetic fields on the faces of the film (at $z=0$ 
and at $-d)$ :

$$
\begin{aligned}
\mathbf{E}_{1 t}(z=0) & =\mathbf{E}_{2 t}(z=0), \quad \mathbf{H}_{1 t}(z=0)=\mathbf{H}_{2 t}(z=0), \\
\mathbf{E}_{2 t}(z=-d) & =\mathbf{E}_{3 t}(z=-d), \quad \mathbf{H}_{2 t}(z=-d)=\mathbf{H}_{3 t}(z=-d),
\end{aligned}
$$

where the subscript " $t$ " in Eqs. (23) stands for the in-plane subvectors. According to Eq. (22), the in-plane subvectors of the magnetic field can be written as $\mathbf{H}_{t}=\mathbf{e}_{z} \times \mathbf{q}_{i} \times \mathbf{E}$.

Using the field representations (17) and (19), we can rewrite the boundary condition at $z=0$ in Eqs. (23) in a more explicit way:

$$
\begin{gathered}
\sum_{\beta=s, p} a_{\beta}^{1}|\beta\rangle=\sum_{\gamma=e, o} a_{\gamma}^{2 \downarrow}|\gamma\rangle+a_{\gamma}^{2 \uparrow}|\gamma\rangle, \\
\mathbf{e}_{z} \times \mathbf{q}_{1+} \times \sum_{\beta=s, p} a_{\beta}^{1}\left|\beta_{1}\right\rangle_{+}=\mathbf{e}_{z} \times \sum_{\gamma=e, o}\left(a_{\gamma}^{2 \downarrow} \mathbf{q}_{\gamma+} \times|\gamma\rangle_{+}+a_{\gamma}^{2 \uparrow} \mathbf{q}_{\gamma-} \times|\gamma\rangle_{-}\right),
\end{gathered}
$$

where $\mathbf{q}_{1,3 \pm}=\left(q_{x}, q_{y}, \pm i q_{1,3 z}\right)^{T}$ and $\mathbf{q}_{\gamma \pm}=\left(q_{x}, q_{y}, \pm i q_{\gamma z}\right)^{T}$. To simplify Eq. (25), let us introduce auxiliary three-dimensional vectors $\left|\beta_{1,3}\right\rangle_{ \pm}^{\prime}$ and $|\gamma\rangle_{ \pm}^{\prime}$

$$
\left|\beta_{1,3}\right\rangle_{ \pm}^{\prime}=-\mathbf{e}_{z} \times \mathbf{q}_{1,3 \pm} \times\left|\beta_{1,3}\right\rangle_{ \pm}, \quad|\gamma\rangle_{ \pm}^{\prime}=-\mathbf{e}_{z} \times \mathbf{q}_{\gamma \pm} \times|\gamma\rangle_{ \pm},
$$

where $\beta=s, p$ and $\gamma=o, e$. Calculating the vector products in Eq. (26), we obtain the following explicit relations for the in-plane two-dimensional subvectors $\left|s_{1,3}\right\rangle^{\prime}$ and $\left|p_{1,3}\right\rangle^{\prime}$ :

$$
\left|s_{1,3}\right\rangle^{\prime}=-Y_{s}^{1,3}|s\rangle, \quad\left|p_{1,3}\right\rangle^{\prime}=-Y_{p}^{1,3}|p\rangle,
$$

being $Y_{\beta}^{1,3}$ the admittances for the $s$ - and $p$-polarized waves:

$$
Y_{s}^{1}=i q_{1 z}, \quad Y_{p}^{1}=\frac{\varepsilon_{1}}{i q_{1 z}}, \quad Y_{s}^{3}=-i q_{3 z}, \quad Y_{p}^{3}=-\frac{\varepsilon_{3}}{i q_{3 z}} .
$$

Since according to Eq. (26), the $z$ component of the three-dimensional vectors $|\gamma\rangle_{ \pm}^{\prime}$ is 0 , we keep the same notation for their two-dimensional in-plane subvectors $|o\rangle_{ \pm}^{\prime}$ and $|e\rangle_{ \pm}^{\prime}$, which read explicitly as

$$
|o\rangle_{ \pm}^{\prime}= \pm i q_{o z}\left[|s\rangle+q_{y} \Delta_{1}|a\rangle\right], \quad|e\rangle_{ \pm}^{\prime}=\frac{\Delta_{2}}{ \pm i q_{e z}}|p\rangle \pm i q_{e z}|b\rangle,
$$

with auxiliary vectors

$$
|a\rangle=\frac{1}{q}\left(\begin{array}{c}
\Delta_{z}+q_{x}^{2} \\
q_{x} q_{y}
\end{array}\right), \quad|b\rangle=\frac{1}{q}\left(\begin{array}{c}
q_{x}\left(c_{2}+1\right) \\
q_{y}
\end{array}\right) .
$$

As a result, using definition (26) and Eqs. (27), we obtain a simplified form of Eq. (25):

$$
\sum_{\beta=s, p} a_{\beta}^{1} Y_{\beta}^{1}|\beta\rangle=\sum_{\gamma=o, e}\left(a_{\gamma}^{2 \downarrow}|\gamma\rangle_{+}^{\prime}+a_{\gamma}^{2 \uparrow}|\gamma\rangle_{-}^{\prime}\right)
$$

If we multiply (24) and (31) by $\langle\beta|$ [here, only the exponential of the bra-vector should be complex conjugated, for example, $\left.\langle s|=\left(\begin{array}{ll}-q_{y} & q_{x}\end{array}\right) e^{-i k_{x} x-i k_{y} y}\right]$ and taking into account that $\left\langle\beta \mid \beta^{\prime}\right\rangle=\delta_{\beta, \beta^{\prime}}$, we get the following system of equations corresponding to the boundary condition at the interface $z=0$ :

$$
\sum_{\gamma=o, e}\left(a_{\gamma}^{2 \downarrow}\langle\beta \mid \gamma\rangle+a_{\gamma}^{2 \uparrow}\langle\beta \mid \gamma\rangle\right)-a_{\beta}^{1}=0, \quad \sum_{\gamma=o, e}\left(a_{\gamma}^{2 \downarrow}\langle\beta \mid \gamma\rangle_{+}^{\prime}+a_{\gamma}^{2 \uparrow}\langle\beta \mid \gamma\rangle_{-}^{\prime}\right)-a_{\beta}^{1} Y_{\beta}^{1}=0 .
$$

Analogously, for the interface $z=-d$, with the help of the auxiliary vectors $|\gamma\rangle_{ \pm}^{\prime}$ we find

$$
\begin{array}{r}
\sum_{\gamma=o, e}\left(a_{\gamma}^{2 \downarrow}\langle\beta \mid \gamma\rangle e^{q_{\gamma z} k_{0} d}+a_{\gamma}^{2 \uparrow}\langle\beta \mid \gamma\rangle e^{-q_{\gamma z} k_{0} d}\right)-a_{\beta}^{3}=0, \\
\sum_{\gamma=o, e}\left(a_{\gamma}^{2 \downarrow}\langle\beta \mid \gamma\rangle_{+}^{\prime} e^{q_{\gamma z} k_{0} d}+a_{\gamma}^{2 \uparrow}\langle\beta \mid \gamma\rangle_{-}^{\prime} e^{-q_{\gamma z} k_{0} d}\right)-a_{\beta}^{3} Y_{\beta}^{3}=0 .
\end{array}
$$


Equations (32) and (33) form a system of eight linear equations with eight unknowns. By defining $\xi^{\gamma \downarrow}=e^{q_{\gamma z} k_{0} d}$ and $\xi^{\gamma \uparrow}=$ $e^{-q_{\gamma z} k_{0} d}$ with $\gamma=o, e$ (for the waves propagating along and opposite to $z$ axis, respectively), we have

$$
\left(\begin{array}{cccccccc}
-1 & 0 & \langle s \mid o\rangle & \langle s \mid o\rangle & \langle s \mid e\rangle & \langle s \mid e\rangle & 0 & 0 \\
0 & -1 & \langle p \mid o\rangle & \langle p \mid o\rangle & \langle p \mid e\rangle & \langle p \mid e\rangle & 0 & 0 \\
-Y_{s}^{1} & 0 & \langle s \mid o\rangle_{+}^{\prime} & \langle s \mid o\rangle_{-}^{\prime} & \langle s \mid e\rangle_{+}^{\prime} & \langle s \mid e\rangle_{-}^{\prime} & 0 & 0 \\
0 & -Y_{p}^{1} & \langle p \mid o\rangle_{+}^{\prime} & \langle p \mid o\rangle_{-}^{\prime} & \langle p \mid e\rangle_{+}^{\prime} & \langle p \mid e\rangle_{-}^{\prime} & 0 & 0 \\
0 & 0 & \langle s \mid o\rangle \xi^{o \downarrow} & \langle s \mid o\rangle \xi^{o \uparrow} & \langle s \mid e\rangle \xi^{\downarrow \downarrow} & \langle s \mid e\rangle \xi^{e \uparrow} & -1 & 0 \\
0 & 0 & \langle p \mid o\rangle \xi^{o \downarrow} & \langle p \mid o\rangle \xi^{o \uparrow} & \langle p \mid e\rangle \xi^{e \downarrow} & \langle p \mid e\rangle \xi^{e \uparrow} & 0 & -1 \\
0 & 0 & \langle s \mid o\rangle_{+}^{\prime} \xi^{o \downarrow} & \langle s \mid o\rangle_{-}^{\prime} \xi^{o \uparrow} & \langle s \mid e\rangle_{+}^{\prime} \xi^{e \downarrow} & \langle s \mid e\rangle_{-}^{\prime} \xi^{e \uparrow} & -Y_{s}^{3} & 0 \\
0 & 0 & \langle p \mid o\rangle_{+}^{\prime} \xi^{o \downarrow} & \langle p \mid o\rangle_{-}^{\prime} \xi^{o \uparrow} & \langle p \mid e\rangle_{+}^{\prime} \xi^{e \downarrow} & \langle p \mid e\rangle_{-}^{\prime} \xi^{e \uparrow} & 0 & -Y_{p}^{3}
\end{array}\right)\left(\begin{array}{c}
a_{s}^{1} \\
a_{p}^{1} \\
a_{o}^{2 \downarrow} \\
a_{o}^{2 \uparrow} \\
a_{e}^{2 \downarrow} \\
a_{e}^{2 \uparrow} \\
a_{s}^{3} \\
a_{p}^{3}
\end{array}\right)=0 .
$$

Using the explicit expressions for the vectors $|s\rangle$ and $|p\rangle,|o\rangle$ and $|e\rangle$, and $|o\rangle_{ \pm}^{\prime},|e\rangle_{ \pm}^{\prime}$, given by Eqs. (16), (20), and (29), respectively, the scalar products in Eq. (34) can be explicitly calculated as

$$
\begin{aligned}
& \langle s \mid o\rangle=\eta_{1}, \\
& \langle p \mid o\rangle=\eta_{2}, \\
& \langle s \mid e\rangle=\eta_{3}, \\
& \langle p \mid e\rangle=\eta_{4}, \\
& \langle s \mid o\rangle_{ \pm}^{\prime}= \pm i q_{o z} \eta_{1}, \\
& \langle p \mid o\rangle_{ \pm}^{\prime}= \pm i q_{o z} \eta_{2} \frac{\varepsilon_{z}}{\Delta_{z}}, \\
& \langle s \mid e\rangle_{ \pm}^{\prime}= \pm i q_{e z} \eta_{3}, \\
& \langle p \mid e\rangle_{ \pm}^{\prime}=\frac{\Delta_{2}}{ \pm i q_{e z}} \pm i q_{e z} \eta_{4},
\end{aligned}
$$

where $\eta_{1}=1-\frac{c_{1} q_{y}^{2}}{q^{2}}, \eta_{2}=\frac{q_{x} q_{y} \Delta_{1}}{q^{2}}\left(\Delta_{z}+q_{x}^{2}+q_{y}^{2}\right), \eta_{3}=\frac{-q_{x} q_{y} c_{2}}{q^{2}}$, and $\eta_{4}=1+\frac{q_{x}^{2} c_{2}}{q^{2}}$.

The homogeneous system (34) has nontrivial solutions only when its determinant equals to zero. The zeros of the determinant yield the dispersion relation for the modes in the biaxial slab. In general, the dispersion relation can be analyzed numerically, but in the limit of a small slab thickness $k_{0} d \ll 1$, as well as in the short-wavelength limit (large values of $q$ ), it can be written in a compact analytical form, as will be shown below, in Secs. V and VI, respectively. Before considering these interesting limits, we will ensure that our dispersion relation analytically reproduces some known examples.

\section{B. Uniaxial slab}

Consider a uniaxial crystal with the axis $C$ pointing along the $z$ axis, $C \| O z$, so that $\varepsilon_{x}=\varepsilon_{y}=\varepsilon_{\perp}$ and $\varepsilon_{z}=\varepsilon_{\|}$. In this case $\Delta_{1}=0$ and $\Delta_{2}=q_{e z}^{2}+\varepsilon_{\perp}$, yielding $c_{1}=c_{2}=0, \eta_{2}=\eta_{3}=0$, and $\eta_{1}=\eta_{4}=1$. Then, the scalar products given by Eq. (35) greatly simplify:

$$
\begin{aligned}
& \langle s \mid o\rangle=\langle p \mid e\rangle=1, \\
& \langle p \mid o\rangle=\langle s \mid e\rangle=\langle p \mid o\rangle_{ \pm}^{\prime}=\langle s \mid e\rangle_{ \pm}^{\prime}=0, \\
& \langle s \mid o\rangle_{ \pm}^{\prime}= \pm i q_{o z}, \\
& \langle p \mid e\rangle_{ \pm}^{\prime}=\frac{\Delta_{2}}{ \pm i q_{e z}} \pm i q_{e z}=\frac{\varepsilon_{\perp}}{ \pm i q_{e z}} .
\end{aligned}
$$

Consequently, the system of equations (34) reduces to

$$
\left(\begin{array}{cccccccc}
-1 & 0 & 1 & 1 & 0 & 0 & 0 & 0 \\
-q_{1 z} & 0 & q_{o z} & -q_{o z} & 0 & 0 & 0 & 0 \\
0 & -1 & \xi^{o \downarrow} & \xi^{o \uparrow} & 0 & 0 & 0 & 0 \\
0 & q_{3 z} & q_{o z} \xi^{o \downarrow} & -q_{o z} \xi^{o \uparrow} & 0 & 0 & 0 & 0 \\
0 & 0 & 0 & 0 & -1 & 0 & 1 & 1 \\
0 & 0 & 0 & 0 & -\frac{\varepsilon_{1}}{q_{z 1}} & 0 & \frac{\Delta_{2}}{q_{e z}} & -\frac{\Delta_{2}}{q_{e z}} \\
0 & 0 & 0 & 0 & 0 & -1 & \xi^{e \downarrow} & \xi^{e^{\uparrow}} \\
0 & 0 & 0 & 0 & 0 & \frac{\varepsilon_{3}}{q_{3 z}} & \frac{\Delta_{2}}{q_{e z}} \xi^{e \downarrow} & -\frac{\Delta_{2}}{q_{e z}} \xi^{e \uparrow}
\end{array}\right)\left(\begin{array}{c}
a_{s}^{1} \\
a_{s}^{3} \\
a_{o}^{2 \downarrow} \\
a_{o}^{2 \uparrow} \\
a_{p}^{1} \\
a_{p}^{3} \\
a_{e}^{2 \downarrow} \\
a_{e}^{2 \uparrow}
\end{array}\right)=0 .
$$


Vanishing of the determinant of the matrix in Eq. (37) results in two separate equations, yielding (after some straightforward algebra) the dispersion of the ordinary and extraordinary modes:

$$
\begin{aligned}
\text { ordinary: } \tanh \left(q_{o z} k_{0} d\right) & =-\frac{q_{o z}\left(q_{1 z}+q_{3 z}\right)}{q_{1 z} q_{3 z}+q_{o z}^{2}}, \\
\text { extraordinary: } \tanh \left(q_{e z} k_{0} d\right) & =-\frac{q_{e z} \varepsilon_{\perp}\left(q_{1 z} \varepsilon_{3}+q_{3 z} \varepsilon_{1}\right)}{q_{1 z} q_{3 z} \varepsilon_{\perp}^{2}+q_{e z}^{2} \varepsilon_{1} \varepsilon_{3}} .
\end{aligned}
$$

\section{Isotropic slab}

The dispersion of the modes in an isotropic slab can be easily derived from the dispersion of the modes in the uniaxial slab, by setting $\varepsilon_{\perp}=\varepsilon_{\|}=\varepsilon_{2}$. By doing so, the $z$ components of the wave vectors $q_{o z}, q_{e z}$ degenerate to $q_{2 z}^{2}=q^{2}-\varepsilon_{2}$ and Eqs. (38) and (39) transform into the well-known expressions for electromagnetic TE and TM modes in a conventional slab waveguide, respectively:

$$
\begin{gathered}
\text { TE: } \tanh \left(q_{2 z} k_{0} d\right)=-\frac{q_{2 z}\left(q_{1 z}+q_{3 z}\right)}{q_{1 z} q_{3 z}+q_{2 z}^{2}}, \\
\mathrm{TM}: \tanh \left(q_{2 z} k_{0} d\right)=-\frac{q_{2 z} \varepsilon_{2}\left(q_{1 z} \varepsilon_{3}+q_{3 z} \varepsilon_{1}\right)}{q_{1 z} q_{3 z} \varepsilon_{2}^{2}+q_{2 z}^{2} \varepsilon_{1} \varepsilon_{3}} .
\end{gathered}
$$

\section{VERY THICK SLABS: SURFACE MODES AT THE BIAXIAL CRYSTAL BOUNDARIES}

Let us consider now another extreme case, assuming that the thickness of the slab tends to infinity, $d \rightarrow \infty$. Then, our dispersion relation should split into two independent dispersion relations describing surface modes at the interfaces between the biaxial crystal and two isotropic media with dielectric permittivities $\varepsilon_{1}$ and $\varepsilon_{3}$. To obtain these dispersion relations in a simple analytical form, we multiply the third and fifth columns of the determinant of the system (34) by $\xi^{o \uparrow}$ and $\xi^{e \uparrow}$, respectively. Then, tending $d \rightarrow \infty$ in the determinant, and assuming that both $q_{o z}$ and $q_{e z}$ have a nonvanishing real part, we see that all the matrix elements proportional to $\xi^{o, e \uparrow}$ (third and fifth elements in the four first rows and fourth and sixth ones in the four last rows) vanish. As a result, the $8 \times 8$ determinant becomes a product of the two determinants $4 \times 4$, each of them describing the surface modes at the 1-2 $(z=0)$ and 2-3 $(z=-d)$ interfaces. Without loss of generality, let us consider only one of these determinants $4 \times 4$, corresponding to the interface 1-2. Zeroing the determinant, we have

$$
\left|\begin{array}{cccc}
-1 & 0 & \langle s \mid o\rangle & \langle s \mid e\rangle \\
0 & -1 & \langle p \mid o\rangle & \langle p \mid e\rangle \\
-Y_{s}^{1} & 0 & \left\langle s \mid o^{\prime}\right\rangle_{-} & \left\langle s \mid e^{\prime}\right\rangle_{-} \\
0 & -Y_{p}^{1} & \left\langle p \mid o^{\prime}\right\rangle_{-} & \left\langle p \mid e^{\prime}\right\rangle_{-}
\end{array}\right|=0 .
$$

Then, using the Gauss method, we can reduce the dimension of the matrix to $2 \times 2$, as

$$
\left|\begin{array}{cc}
Y_{s}^{1}\langle s \mid o\rangle-\left\langle s \mid o^{\prime}\right\rangle_{-} & Y_{s}^{1}\langle s \mid e\rangle-\left\langle s \mid e^{\prime}\right\rangle_{-} \\
Y_{p}^{1}\langle p \mid o\rangle-\left\langle p \mid o^{\prime}\right\rangle_{-} & Y_{p}^{1}\langle p \mid e\rangle-\left\langle p \mid e^{\prime}\right\rangle_{-}
\end{array}\right|=0 .
$$

To write the dispersion relation in a compact form, we express $\varepsilon_{z}$ from Frensel's equation for biaxial slabs (6) as a function of $q_{o z}$ :

$$
\varepsilon_{z}=\frac{\varepsilon_{x} \varepsilon_{y} q^{2}+\left(q_{o z}^{2}-q^{2}\right)\left(\varepsilon_{x} q_{x}^{2}+\varepsilon_{y} q_{y}^{2}\right)}{q_{o z}^{2}\left(\varepsilon_{x}+\varepsilon_{y}+q_{o z}^{2}-q^{2}\right)+\varepsilon_{x} \varepsilon_{y}-\varepsilon_{x} q_{x}^{2}-\varepsilon_{y} q_{y}^{2}} .
$$

Then, using the identities $q^{2}=\varepsilon_{1}+q_{1 z}^{2}$ and $q^{2}=q_{x}^{2}+q_{y}^{2}$, we substitute $\varepsilon_{z}$ from Eq. (44) into the expressions for $Y_{s, p}^{1}$ in Eq. (28) and scalar products given by Eq. (35), appearing in the elements of the matrix in Eq. (43). After some algebraic operations, Eq. (43) reproduces the dispersion relation for surface waves on boundaries of biaxial crystals, derived in Ref. [21]:

$$
\begin{aligned}
& \left(q_{1 z}+q_{o z}\right)\left(q_{1 z}+q_{e z}\right)\left(\varepsilon_{x} \varepsilon_{y}-\varepsilon_{x} q_{x}^{2}-\varepsilon_{y} q_{y}^{2}-\varepsilon_{1} q_{o z} q_{e z}\right) \\
& -q_{o z} q_{e z}\left(\varepsilon_{1}-\varepsilon_{x}\right)\left(\varepsilon_{1}-\varepsilon_{y}\right)=0 .
\end{aligned}
$$

\section{A. Uniaxial crystal with the axis perpendicular to the interface}

Consider a uniaxial crystal with the axis $C$ along the $z$ axis, thus directed perpendicularly to the interface of the crystal. Defining, as before, $\varepsilon_{x}=\varepsilon_{y}=\varepsilon_{\perp}$ and $\varepsilon_{z}=\varepsilon_{\|}$, and taking into account that, according to Eq. (12), $\varepsilon_{\perp}=q^{2}-q_{o z}^{2}$, Eq. (45) simplifies as

$$
\begin{gathered}
q_{o z}\left(q_{1 z}+q_{o z}\right)\left(q_{1 z}+q_{e z}\right)\left(\varepsilon_{\perp} q_{o z}+\varepsilon_{1} q_{e z}\right) \\
+q_{o z} q_{e z}\left(q_{1 z}+q_{o z}\right)^{2}\left(q_{1 z}-q_{o z}\right)^{2}=0 .
\end{gathered}
$$

Since $\operatorname{Re}\left(q_{o z}\right)>0$ and $\operatorname{Re}\left(q_{1 z}\right)>0$, and therefore $q_{o z}\left(q_{z}+q_{o z}\right) \neq 0$, we can divide Eq. (46) by $q_{o z}\left(q_{z}+q_{o z}\right)$. Then, it transforms to

$$
q_{e z} \varepsilon_{1}\left(q_{o z}+q_{e z}\right)+q_{1 z} \varepsilon_{\perp}\left(q_{o z}+q_{e z}\right)=0 .
$$

Assuming that $q_{e z}+q_{o z} \neq 0$, we obtain the dispersion relation for surface waves on a boundary of a uniaxial crystal:

$$
\frac{q_{e z}}{\varepsilon_{\perp}}+\frac{q_{1 z}}{\varepsilon_{1}}=0
$$

where $q_{1 z}=\sqrt{q^{2}-\varepsilon_{1}}$ and $q_{e z}=\sqrt{\frac{\varepsilon_{\perp}}{\varepsilon_{\|}} q^{2}-\varepsilon_{\perp}}$. Deriving $q$ from this equation, the dispersion relation takes the wellknown form (see e.g. Ref. [28])

$$
q=\sqrt{\varepsilon_{1} \varepsilon_{\|} \frac{\varepsilon_{1}-\varepsilon_{\perp}}{\varepsilon_{1}^{2}-\varepsilon_{\|} \varepsilon_{\perp}}}
$$

In the isotropic case $\varepsilon_{\|}=\varepsilon_{\perp}=\varepsilon$, Eq. (48) simplifies to the dispersion relation for surface waves at the interface between two isotropic media:

$$
q=\sqrt{\frac{\varepsilon_{1} \varepsilon}{\varepsilon_{1}+\varepsilon}}
$$

\section{B. Uniaxial case with in-plane crystal axis}

Consider a uniaxial crystal with the $C$ axis along the $y$ axis, thus lying in the plane of the interface of the crystal. Redefining here $\varepsilon_{x}=\varepsilon_{z}=\varepsilon_{\perp}$ and $\varepsilon_{y}=\varepsilon_{\|}$, Eq. (7) for $q_{o, e z}$ transforms to [similar to Eq. (12)]

$$
q_{o z}^{2}=q^{2}-\varepsilon_{\perp}, \quad \varepsilon_{\perp} q_{e z}^{2}=\varepsilon_{\perp} q_{x}^{2}-\varepsilon_{\|} q_{y}^{2}-\varepsilon_{\perp} \varepsilon_{\|} .
$$

Substituting the expression for $q_{e z}$ from Eq. (51) into Eq. (45) and dividing it by $q_{e z}$, we get the famous dispersion relation 


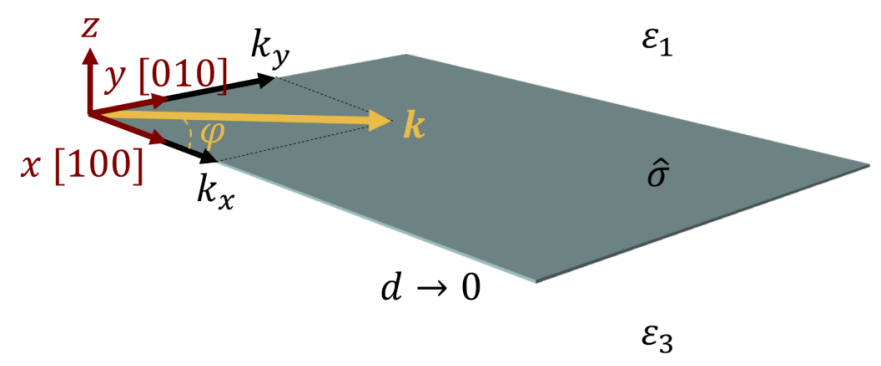

FIG. 2. Schematics of the ultrathin crystal slab.

for Dyakonov surface waves [19]:

$$
\begin{gathered}
\left(q_{1 z}+q_{o z}\right)\left(q_{1 z}+q_{e z}\right)\left(\varepsilon_{1} q_{o z}+\varepsilon_{\perp} q_{e z}\right) \\
+q_{o z}\left(\varepsilon_{1}-\varepsilon_{\perp}\right)\left(\varepsilon_{1}-\varepsilon_{\|}\right)=0 .
\end{gathered}
$$

\section{ULTRATHIN SLAB LIMIT}

Recently, polaritons in ultrathin slabs and monolayers (for instance, plasmon polaritons in a monolayer graphene [29] or hyperbolic phonon polaritons in thin slabs of polar dielectrics, such as $h$-BN [30]) have attracted particularly high attention. Therefore, the limit of a vanishing slab thickness $d \rightarrow 0$ is of a great practical interest. Let us illustrate how our general dispersion relation, given by the determinat of the system (34), can be simplified for ultrathin slabs. Analogously to the methodology used for isotropic slabs [31], we can approximate the slab of a finite thickness by a two-dimensional (2D) conductive sheet, with the effective conductivity $\hat{\sigma}$ given by $\hat{\sigma}=\frac{\omega d \hat{\varepsilon}}{4 \pi i}$ (see Fig. 2). To that end, let us assume that all the components of the tensor $\hat{\varepsilon}$ are large, i.e., $\left|\varepsilon_{i}\right| \gg 1(i=$ $x, y, z)$.

Then, retaining in Eq. (7) the first nonvanishing terms depending upon $q_{x}$ and $q_{y}$ in the expressions for the normalized $z$ components of the wave vectors $q_{o, e z}$, Eq. (7) can be greatly simplified:

$$
\begin{aligned}
q_{o, e z}^{2}= & \frac{1}{2}\left\{-\left(\varepsilon_{x}+\varepsilon_{y}\right)+\frac{\varepsilon_{x}+\varepsilon_{z}}{\varepsilon_{z}} q_{x}^{2}+\frac{\varepsilon_{y}+\varepsilon_{z}}{\varepsilon_{z}} q_{y}^{2}\right\} \\
& \pm \frac{1}{2}\left\{\varepsilon_{x}-\varepsilon_{y}+\frac{\varepsilon_{z}-\varepsilon_{x}}{\varepsilon_{z}} q_{x}^{2}-\frac{\varepsilon_{z}-\varepsilon_{y}}{\varepsilon_{z}} q_{y}^{2}\right\}
\end{aligned}
$$

where + and - should be taken for the labels $o$ and $e$, respectively. Equations (53) then further simplify to

$$
\begin{aligned}
& q_{o z}^{2}=-\varepsilon_{y}+q_{x}^{2}+\frac{\varepsilon_{y}}{\varepsilon_{z}} q_{y}^{2}, \\
& q_{e z}^{2}=-\varepsilon_{x}+q_{y}^{2}+\frac{\varepsilon_{x}}{\varepsilon_{z}} q_{x}^{2} .
\end{aligned}
$$

Using Eq. (54), we find $c_{1}=-\frac{\varepsilon_{z}}{q_{y}^{2}}$ and $c_{2}=-1$, so that the scalar products (35) can be written as

$$
\begin{aligned}
& \langle s \mid o\rangle=\frac{q_{x}^{2}}{q^{2}} \\
& \langle p \mid o\rangle=\frac{q_{x} q_{y}}{q^{2}}, \\
& \langle s \mid e\rangle=\varepsilon_{z} \frac{\varepsilon_{x}-\varepsilon_{y}}{\varepsilon_{x}-\varepsilon_{z}} \frac{q_{y}}{q_{x} q^{2}}, \\
& \langle p \mid e\rangle=-\varepsilon_{z} \frac{\varepsilon_{x}-\varepsilon_{y}}{\varepsilon_{x}-\varepsilon_{z}} \frac{1}{q^{2}}, \\
& \langle s \mid o\rangle_{ \pm}^{\prime}= \pm i q_{o z}\langle s \mid o\rangle, \\
& \langle p \mid o\rangle_{ \pm}^{\prime}= \pm i q_{o z}\langle p \mid o\rangle, \\
& \langle s \mid e\rangle_{ \pm}^{\prime}= \pm i q_{e z}\langle s \mid e\rangle, \\
& \langle p \mid e\rangle_{ \pm}^{\prime}= \pm i q_{e z}\langle p \mid e\rangle .
\end{aligned}
$$

Additionally, assuming the small thickness of the slab, we can simplify the elements of the matrix in Eq. (34) by expanding the exponentials $\xi^{\gamma}$ into the Taylor series in $k_{0} d$ and retaining the first nonvanishing terms. We have $\xi^{\gamma \downarrow}=e^{q_{\gamma z} k_{0} d}=$ $1+q_{\gamma z} k_{0} d$ and $\xi^{\gamma \uparrow}=e^{-q_{\gamma z} k_{0} d}=1-q_{\gamma z} k_{0} d$. To simplify the determinant of the system (34), we sum up its third and fifth columns with the fourth and sixth columns, respectively, and then subtract the fourth and sixth columns (both multiplied by the factor $\frac{1}{2}$ ), from the third and fifth columns, respectively. Then using row operations, we eliminate two first and two last columns in the obtained determinant by the Gauss method: we multiply first (second) row to $Y_{s}^{1}\left(Y_{p}^{1}\right)$ and subtract it from the third (fourth) row and analogously for fifth (sixth) and seventh (eighth) rows. As a result, we get the following equation:

$$
\left|\begin{array}{cccc}
i\langle s \mid o\rangle & -Y_{s}^{1}\langle s \mid o\rangle & i\langle s \mid e\rangle & -Y_{s}^{1}\langle s \mid e\rangle \\
i\langle p \mid o\rangle & -Y_{p}^{1}\langle p \mid o\rangle & i\langle p \mid e\rangle & -Y_{p}^{1}\langle p \mid e\rangle \\
-Y_{s}^{3} k_{0} d\langle s \mid o\rangle & \left(2 \alpha_{y}+Y_{s}^{1}-Y_{s}^{3}\right)\langle s \mid o\rangle & -Y_{s}^{3} k_{0} d\langle s \mid e\rangle & \left(2 \alpha_{x}+Y_{s}^{1}-Y_{s}^{3}\right)\langle s \mid e\rangle \\
-Y_{p}^{3} k_{0} d\langle p \mid o\rangle & \left(2 \alpha_{y}+Y_{p}^{1}-Y_{p}^{3}\right)\langle p \mid o\rangle & -Y_{p}^{3} k_{0} d\langle p \mid e\rangle & \left(2 \alpha_{x}+Y_{p}^{1}-Y_{p}^{3}\right)\langle p \mid e\rangle
\end{array}\right|=0,
$$

where $\alpha_{x, y}=\frac{2 \pi \sigma_{x, y}}{c}=\frac{k_{0} d \varepsilon_{x, y}}{2 i}$ are the normalized 2D effective conductivity components. Using the smallness of $k_{0} d$, on the one hand, and the assumed large values of the components of the tensor $\hat{\varepsilon}$, on the other hand, the determinant (56) can be further significantly simplified. Namely, a more detailed analysis (which we omit here) shows that the elements proportional to $k_{0} d$ (the first and third elements of the third and fourth rows) yield the contribution of a higher order of smallness and thus can be neglected. As a result, the determinant (56) factorizes into a product of the two determinants of the submatrices $2 \times 2$ :

$$
\left|\begin{array}{cc}
\langle s \mid o\rangle & \langle s \mid e\rangle \\
\langle p \mid o\rangle & \langle p \mid e\rangle
\end{array}\right|\left|\begin{array}{cc}
\left(2 \alpha_{y}+Y_{s}^{1}-Y_{s}^{3}\right) q_{x} & \left(2 \alpha_{x}+Y_{s}^{1}-Y_{s}^{3}\right) q_{y} \\
\left(2 \alpha_{y}+Y_{p}^{1}-Y_{p}^{3}\right) q_{y} & -\left(2 \alpha_{x}+Y_{p}^{1}-Y_{p}^{3}\right) q_{x}
\end{array}\right|=0 .
$$


(a)

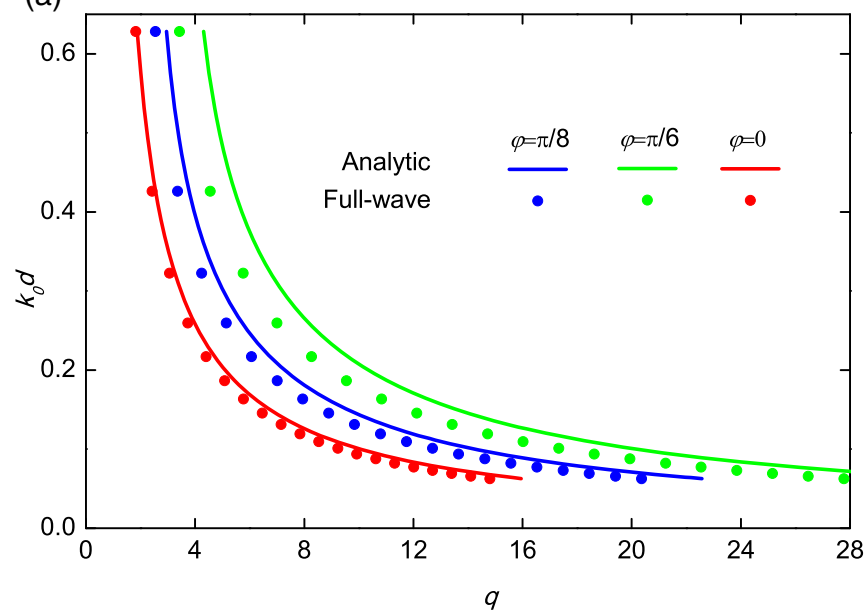

(b)

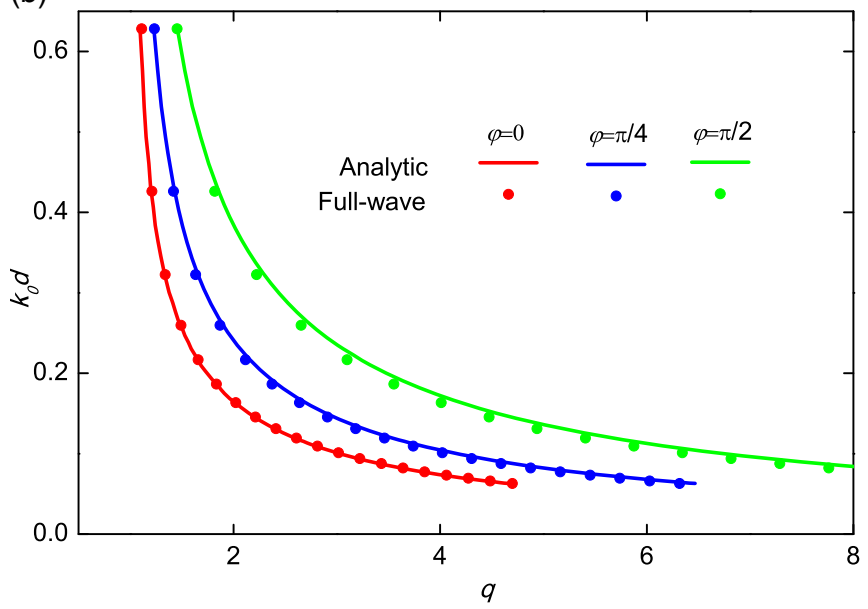

FIG. 3. Comparison of the dispersion relation given by Eq. (58) and by full-wave numerical simulations (COMSOL MULTIPHYSICS). In both panels, $\varepsilon_{1}=\varepsilon_{3}=1$. Permittivity tensor components: (a) $\varepsilon_{x}=-2, \varepsilon_{y}=2, \varepsilon_{z}=2$. (b) $\varepsilon_{x}=-7, \varepsilon_{y}=-3, \varepsilon_{z}=2$.

Taking into account that the first determinant in Eq. (57) gives $-\frac{\varepsilon_{z}}{q^{2}} \frac{\varepsilon_{x}-\varepsilon_{y}}{\varepsilon_{x}-\varepsilon_{z}} \neq 0$, the dispersion relation is given by the vanishing of the the second determinant:

$$
\left\{\alpha_{x} q_{y}^{2}+\alpha_{y} q_{x}^{2}+\frac{q_{x}^{2}+q_{y}^{2}}{2}\left(i q_{1 z}+i q_{3 z}\right)\right\}\left\{\alpha_{x} q_{x}^{2}+\alpha_{y} q_{y}^{2}+\frac{q_{x}^{2}+q_{y}^{2}}{2}\left(\frac{\varepsilon_{1}}{i q_{1 z}}+\frac{\varepsilon_{3}}{i q_{3 z}}\right)\right\}=q_{x}^{2} q_{y}^{2}\left(\alpha_{x}-\alpha_{y}\right)^{2} .
$$

This dispersion relation, written for biaxial slabs of small but nonzero thickness (the effective conductivities $\alpha_{x, y}$ are thickness dependent), has been used for the analysis of hyperbolic phonon polaritons in thin slabs of $\alpha-\mathrm{MoO}_{3}$ [16]. Nevertheless, to our knowledge, it has not been consistently derived for a nonvanishing slab thickness up to now. For 2D anisotropic sheets (of zero thickness) Eq. (58) is exact and had been reported in Refs. [32,33]. As expected, the asymptotes of the dispersion relation (58) $\left(q_{x, y} \rightarrow \infty\right)$ coincide with the ones following from the Fresnel equations [compare with Eq. (11)]:

$$
\frac{q_{x}}{q_{y}}=\sqrt{-\frac{\alpha_{y}}{\alpha_{x}}}=\sqrt{-\frac{\varepsilon_{y}}{\varepsilon_{x}}} .
$$

In case of an isotropic 2D sheet $\alpha_{x}=\alpha_{y}=\alpha$ and Eq. (58) splits into two independent equations describing the dispersion of the TE and TM modes [34] propagating along the sheet:

$$
\begin{aligned}
\text { TE }: & q_{1 z}+q_{3 z}-2 i \alpha=0, \\
\mathrm{TM}: & \frac{\varepsilon_{1}}{q_{1 z}}+\frac{\varepsilon_{3}}{q_{3 z}}+2 i \alpha=0 .
\end{aligned}
$$

To demonstrate the validity of the simplified dispersion relation (58), we compare in Fig. 3 the refractive indices of a mode found from (58) (solid curves) to those found from full-wave simulations (points). Figures 3(a) and 3(b) represent the result for two different illustrative set of parameters: in Fig. 3(a) only one of the in-plane dielectric permittivities is negative, $\varepsilon_{x}<0$ (while $\varepsilon_{y}, \varepsilon_{z}>0$ ), and in Fig. 3(b) both in-plane permittivities are negative, $\varepsilon_{x}, \varepsilon_{y}<0$ (while $\varepsilon_{z}>0$ ). Both in Figs. 3(a) and 3(b) the propagation of the mode at different angles $\varphi$ (see Fig. 2) is considered. As expected, the agreement between the analytical approximation and rigorous numeric simulations improves for smaller values of $k_{0} d$, although in Fig. 3(b) the agreement is good in the whole shown range of $k_{0} d$. Impressively, the agreement between the analytical and numerical results is in general excellent for all the shown propagation directions, even though neither $k_{0} d$ is very small nor the values of $\varepsilon_{x}, \varepsilon_{y}$, and $\varepsilon_{z}$ are very large, as it was initially assumed for the derivation of (58).

\section{LIMIT OF A LARGE REFRACTIVE INDEX OF THE MODES}

The general dispersion relation given by the zeroing of the determinant in Eq. (34) can be also greatly simplified under the assumption of large refractive indices of the modes $|q| \gg 1$. Such simplification is similar to the one made for the dispersion of the modes in uniaxial crystal slabs [14]. For large $q$ the expressions for the $z$ components of the wave vectors inside the slab can be approximated as

$$
q_{o z}^{2}=q^{2}-\frac{\varepsilon_{x} \varepsilon_{z} q_{y}^{2}+\varepsilon_{y} \varepsilon_{z} q_{x}^{2}-\varepsilon_{x} \varepsilon_{y} q^{2}}{\varepsilon_{z} q^{2}-\varepsilon_{x} q_{x}^{2}-\varepsilon_{y} q_{y}^{2}}, \quad q_{e z}^{2}=\frac{\varepsilon_{x}}{\varepsilon_{z}} q_{x}^{2}+\frac{\varepsilon_{y}}{\varepsilon_{z}} q_{y}^{2},
$$

where we have retained the second-order (the highest-order) term in $q$ both in $q_{e z}^{2}$ and $q_{o z}^{2}$, as well as the zeroth-order term in the expression for $q_{o z}^{2}$, to avoid uncertainty in $\Delta_{1}$ (since $\Delta_{x}^{o}=q_{x}^{2}$ and $\Delta_{z}=q_{o z}^{2}=q^{2}$, we have $\left.\Delta_{1}=\frac{0}{0}\right)$. Substituting 
the Eqs. (61) into Eqs. (10) and (21), we have

$$
\begin{aligned}
& \Delta_{1}=\frac{1}{q_{o z}^{2}} \frac{\varepsilon_{x}-\varepsilon_{y}}{\varepsilon_{z}-\varepsilon_{x}}, \quad \Delta_{2}=q_{e z}^{2}, \\
& c_{1}=\frac{\varepsilon_{x}-\varepsilon_{y}}{\varepsilon_{x}-\varepsilon_{z}}, \quad c_{2}=\frac{\varepsilon_{y}-\varepsilon_{x}}{q_{e z}^{2}-q_{o z}^{2}},
\end{aligned}
$$

where we have neglected all small amendments in the expressions for each constant. Then, we obtain the simplified expressions for the scalar products (35):

$$
\begin{aligned}
& \langle s \mid o\rangle=\frac{\varepsilon_{z}}{\varepsilon_{x}-\varepsilon_{z}} \frac{q_{e z}^{2}-q_{o z}^{2}}{q_{o z}^{2}}, \\
& \langle p \mid o\rangle=\frac{q_{x} q_{y}}{q_{o z}^{2}} \frac{\varepsilon_{x}-\varepsilon_{y}}{\varepsilon_{x}-\varepsilon_{z}},
\end{aligned}
$$

$$
\mid \begin{array}{cc}
\left\langle s \mid o^{\prime}\right\rangle_{+} & -Y_{s}^{1}\langle s \mid o\rangle \\
\left\langle p \mid o^{\prime}\right\rangle_{+} & -Y_{p}^{1}\langle p \mid o\rangle \\
\left\langle s \mid o^{\prime}\right\rangle_{+} C_{o}-Y_{s}^{3}\langle s \mid o\rangle S_{o} & \left\langle s \mid o^{\prime}\right\rangle_{+} S_{o}-Y_{s}^{3}\langle s \mid o\rangle C_{o} \\
\left\langle p \mid o^{\prime}\right\rangle_{+} C_{o}-Y_{p}^{3}\langle p \mid o\rangle S_{o} & \left\langle p \mid o^{\prime}\right\rangle_{+} S_{o}-Y_{p}^{3}\langle p \mid o\rangle C_{o}
\end{array}
$$

where, for compactness, we have introduced abbreviated notations for the hyperbolic functions:

$$
C_{o, e}=\cosh \left(q_{o, e z} k_{0} d\right), \quad S_{o, e}=\sinh \left(q_{o, e z} k_{0} d\right) .
$$

Let us notice that the matrix elements containing either $\left\langle s \mid o^{\prime}\right\rangle_{+}$ or $Y_{s}^{1,3}\langle s \mid o\rangle$ are of order $\sim q$, while the other matrix elements are of order $\sim \frac{1}{q}$, thus being much smaller in magnitude. Consequently, we can neglect the third and fourth elements of the first and third rows (the contribution of these elements to the determinant is of the second and fourth order of smallness in $\frac{1}{q}$ ). As a result, the determinant in Eq. (64) factorizes into a product of two determinants of the submatrices $2 \times 2$, so that Eq. (64) splits into the two following equations:

$$
\begin{aligned}
& \left|\begin{array}{cc}
\left\langle s \mid o^{\prime}\right\rangle_{+} & -Y_{s}^{1}\langle s \mid o\rangle \\
\left\langle s \mid o^{\prime}\right\rangle_{+} C_{o}-Y_{s}^{3}\langle s \mid o\rangle S_{o} & \left\langle s \mid o^{\prime}\right\rangle_{+} S_{o}-Y_{s}^{3}\langle s \mid o\rangle C_{o}
\end{array}\right|=0, \\
& \left|\begin{array}{cc}
\left\langle p \mid e^{\prime}\right\rangle_{+} & -Y_{p}^{1}\langle p \mid e\rangle \\
\left\langle p \mid e^{\prime}\right\rangle_{+} C_{e}-Y_{p}^{3}\langle p \mid e\rangle S_{e} & \left\langle p \mid e^{\prime}\right\rangle_{+} S_{e}-Y_{p}^{3}\langle p \mid e\rangle C_{e}
\end{array}\right|=0 .
\end{aligned}
$$

Simplifying all the admittances as $Y_{p}^{1}=\frac{\varepsilon_{1}}{q_{1 z}} \approx \frac{\varepsilon_{1}}{q} \approx \frac{\varepsilon_{1}}{q_{o z}}$, $Y_{p}^{3}=-\frac{\varepsilon_{3}}{q_{3 z}} \approx-\frac{\varepsilon_{3}}{q} \approx-\frac{\varepsilon_{3}}{q_{o z}}$, and $Y_{s}^{1} \approx-Y_{s}^{3} \approx i q \approx i q_{o z}$, we can easily calculate both determinants (66). Vanishing of the first determinant does not give any physically reasonable solutions since

$$
-\frac{2 \varepsilon_{z}^{2}\left(q_{e z}^{2}-q_{o z}^{2}\right)^{2} e^{q_{o z} k_{0} d}}{\left(\varepsilon_{x}-\varepsilon_{z}\right)^{2} q_{o z}^{2}} \neq 0 .
$$

Therefore, the dispersion relation follows from the vanishing of the second determinant in Eq. (66):

$$
\tanh \left(q_{e z} k_{0} d\right)=-\frac{\left(\varepsilon_{1}+\varepsilon_{3}\right) \varepsilon_{z} q_{o z} q_{e z}}{\varepsilon_{1} \varepsilon_{3} q_{o z}^{2}+\varepsilon_{z}^{2} q_{e z}^{2}}
$$

$$
\begin{aligned}
& \langle s \mid e\rangle=\frac{q_{x} q_{y}}{q_{o z}^{2}} \frac{\varepsilon_{x}-\varepsilon_{y}}{q_{e z}^{2}-q_{o z}^{2}}, \\
& \langle p \mid e\rangle=1, \\
& \left\langle s \mid o^{\prime}\right\rangle_{ \pm}= \pm i q_{o z}\langle s \mid o\rangle, \\
& \left\langle p \mid o^{\prime}\right\rangle_{ \pm}= \pm i \varepsilon_{z} \frac{q_{x} q_{y}}{q_{o z}^{3}} \frac{\varepsilon_{x}-\varepsilon_{y}}{\varepsilon_{z}-\varepsilon_{x}}, \\
& \left\langle s \mid e^{\prime}\right\rangle_{ \pm}= \pm i q_{e z}\langle s \mid e\rangle, \\
& \left\langle p \mid e^{\prime}\right\rangle_{ \pm}=\mp i q_{e z} \frac{\varepsilon_{z}}{q_{o z}^{2}} .
\end{aligned}
$$

To simplify the matrix (34) and to eliminate the two first and two last columns, we use the same column and row operations as in Sec. V and obtain the following equation (containing the $4 \times 4$ matrix):

$$
\begin{array}{cc}
\left\langle s \mid e^{\prime}\right\rangle_{+} & -Y_{s}^{1}\langle s \mid e\rangle \\
\left\langle p \mid e^{\prime}\right\rangle_{+} & -Y_{p}^{1}\langle p \mid e\rangle \\
\left\langle s \mid e^{\prime}\right\rangle_{+} C_{e}-Y_{s}^{3}\langle s \mid e\rangle S_{e} & \left\langle s \mid e^{\prime}\right\rangle_{+} S_{e}-Y_{s}^{3}\langle s \mid e\rangle C_{e} \\
\left\langle p \mid e^{\prime}\right\rangle_{+} C_{e}-Y_{p}^{3}\langle p \mid e\rangle S_{e} & \left\langle p \mid e^{\prime}\right\rangle_{+} S_{e}-Y_{p}^{3}\langle p \mid e\rangle C_{e}
\end{array} \mid=0,
$$

To write Eq. (68) in a convenient form, let us define

$$
\rho=i \sqrt{\frac{\varepsilon_{z} q^{2}}{\varepsilon_{x} q_{x}^{2}+\varepsilon_{y} q_{y}^{2}}}=i \sqrt{\frac{\varepsilon_{z}}{\varepsilon_{x} \cos ^{2} \varphi+\varepsilon_{y} \sin ^{2} \varphi}},
$$

where $\varphi$ is the angle between the $x$ axis and the in-plane component of wave vector. Then, using Eq. (61) (neglecting here the second term in $q_{o z}^{2}$ ), Eq. (68) can be written as

$$
\tan \left(\frac{q k_{0} d}{\rho}\right)=\frac{\rho \frac{\varepsilon_{1}+\varepsilon_{3}}{\varepsilon_{z}}}{1-\frac{\rho^{2} \varepsilon_{1} \varepsilon_{3}}{\varepsilon_{z}^{2}}} .
$$

Taking into account that $\arctan \left(\frac{x+y}{1-x y}\right)=\arctan (x)+$ $\arctan (y)$, we get a simple expression for the normalized in-plane wave vector $q$ in the biaxial slab in the short-wavelength limit $q \gg 1$ :

$$
\begin{array}{r}
q=\frac{\rho}{k_{0} d}\left[\arctan \left(\frac{\varepsilon_{1} \rho}{\varepsilon_{z}}\right)+\arctan \left(\frac{\varepsilon_{3} \rho}{\varepsilon_{z}}\right)+\pi l\right], \\
l=0,1,2 \ldots .
\end{array}
$$

We can verify that Eq. (71) transforms into the dispersion of modes in a uniaxial slab (with the axis $C$ along the $z$ axis), setting $\varepsilon_{x}=\varepsilon_{y}=\varepsilon_{\perp}$ and $\varepsilon_{z}=\varepsilon_{\|}$. Then, defining $\psi=$ $-\rho=-i \sqrt{\frac{\varepsilon_{\|}}{\varepsilon_{\perp}}}$, and taking into account that $\frac{\varepsilon_{1,3} \rho}{\varepsilon_{\|}}=\frac{\varepsilon_{1,3}}{\psi \varepsilon_{\perp}}$, we reproduce the dispersion relation, used for the analysis of hyperbolic phonon polaritons in $h$-BN crystal slabs [14]:

$$
\begin{array}{r}
q=-\frac{\psi}{k_{0} d}\left[\arctan \left(\frac{\varepsilon_{1}}{\psi \varepsilon_{\perp}}\right)+\arctan \left(\frac{\varepsilon_{3}}{\psi \varepsilon_{\perp}}\right)+\pi l\right], \\
l=0,1,2 \ldots .
\end{array}
$$

The same results can be straightforwardly derived from the exact Eq. (39), in the limit of large $q$. Equation (71) also reduces 
(a)

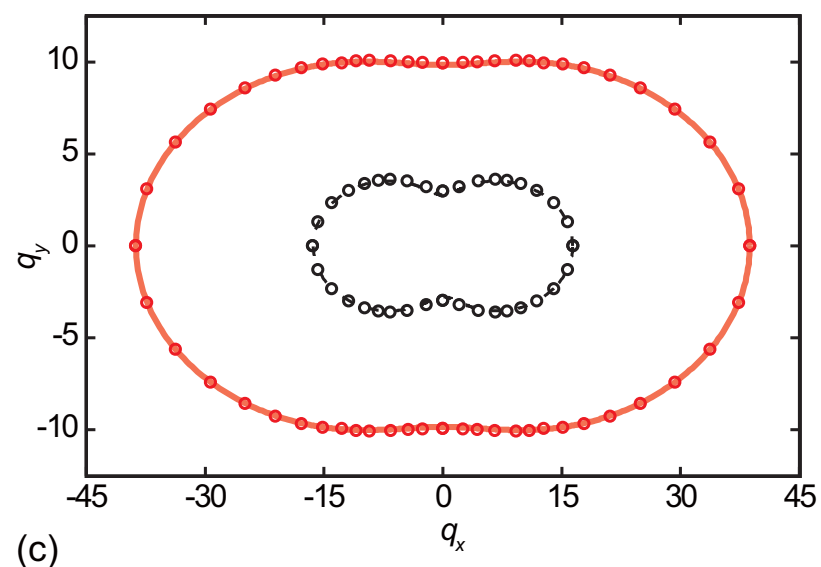

(c)

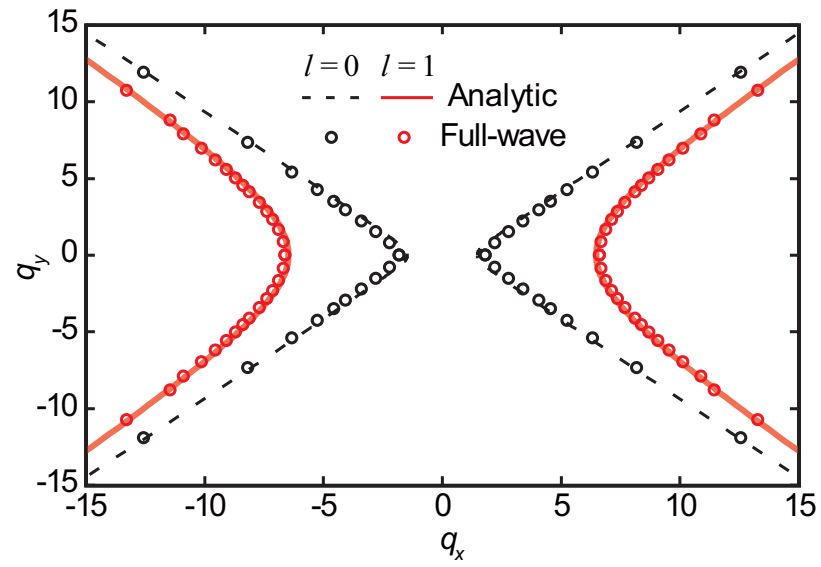

(b)

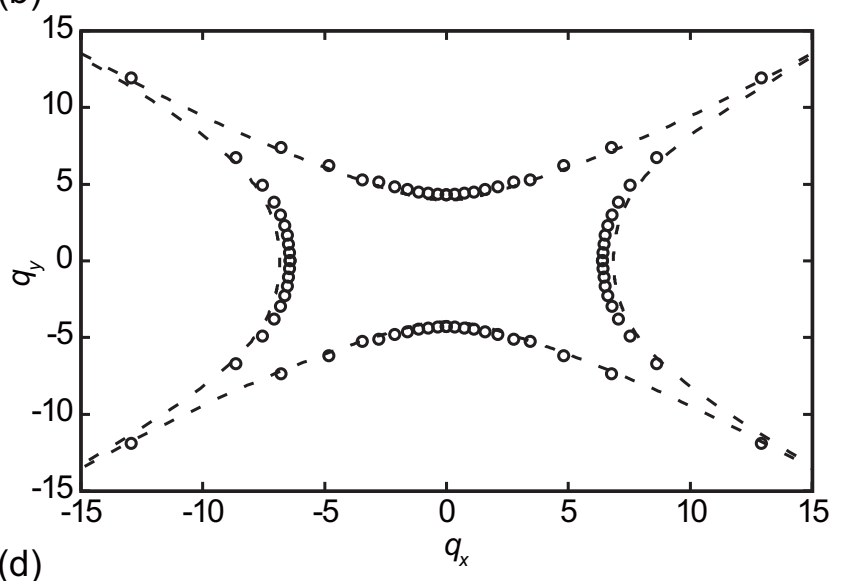

(d)

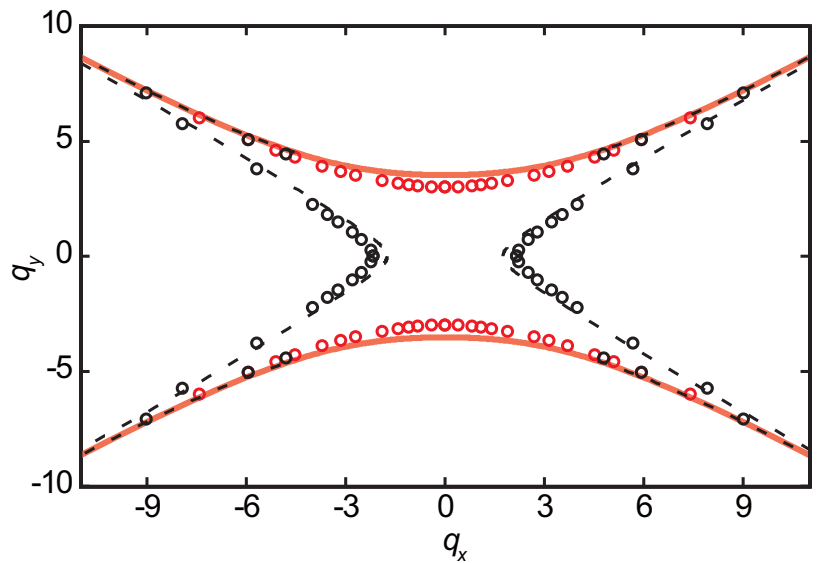

FIG. 4. Comparison between the isofrequency curves of the modes in a biaxial crystal slab. The isofrequency curves found according to Eq. (71) are plotted by the discontinuous black curves $(l=0)$ and continuous red curves $(l=1)$, while those found from the full-wave simulations are plotted by the black and red dots, respectively. In all panels $\lambda=1 \mu \mathrm{m}, d=100 \mathrm{~nm}$, and $\varepsilon_{1}=\varepsilon_{3}=1$. Permittivity tensor components: (a) $\varepsilon_{x}=-0.1, \varepsilon_{y}=-1, \varepsilon_{z}=2$. (b) $\varepsilon_{x}=-0.1, \varepsilon_{y}=-1, \varepsilon_{z}=-2$. (c) $\varepsilon_{x}=-2, \varepsilon_{y}=2$, $\varepsilon_{z}=2$. (d) $\varepsilon_{x}=-2$, $\varepsilon_{y}=2$, $\varepsilon_{z}=-2$.

to Eq. (72) when the propagation of the mode coincides either with the $x$ axis (in this case we should set $\varepsilon_{x}=\varepsilon_{\perp}$ and $\varepsilon_{z}=\varepsilon_{\|}$) or with the $y$ axis (in this case we should set $\varepsilon_{y}=\varepsilon_{\perp}$ and $\varepsilon_{z}=$ $\left.\varepsilon_{\|}\right)$. In the two latter particular cases, anisotropic polaritons in $\alpha-\mathrm{MoO}_{3}$ slabs were studied via Eq. (72) in Ref. [18].

To verify the validity of our analytical approximation, we compare the isofrequency curves obtained from Eq. (71) with those obtained from full-wave electromagnetic simulations. As an example, we take the slab thickness $d=100 \mathrm{~nm}$ and the free-space wavelength $\lambda=1 \mu \mathrm{m}$. We consider the following four different combinations of the (purely real) permittivity tensor components:

$$
\begin{aligned}
& \text { (a) } \varepsilon_{x}<0, \varepsilon_{y}<0, \varepsilon_{z}>0, \\
& \text { (b) } \varepsilon_{x}<0, \varepsilon_{y}<0, \varepsilon_{z}<0, \\
& \text { (c) } \varepsilon_{x}<0, \varepsilon_{y}>0, \varepsilon_{z}>0, \\
& \text { (d) } \varepsilon_{x}<0, \varepsilon_{y}>0, \varepsilon_{z}<0 .
\end{aligned}
$$

Figure 4 shows the isofrequency curves extracted from both Eq. (71) (red and black curves) and full-wave numeric simulations (dots). For the parametric sets (a), (c), (d) corresponding to the volume modes, the isofrequency curves for the two lowest modes are shown: $l=0$ (black discontinuous curve and black dots) and $l=1$ (red continuous curve and red dots). In contrast, for the set of parameters (b), the mode exponentially decays inside and outside the slab (so that it has a surface wave character) and therefore only the solution with $l=0$ makes sense. In all panels of Fig. 4 we see an excellent agreement between the numeric simulations and analytical approximations for large $q(q \gtrsim 10)$ and even very reasonable agreement for $q$ comparable to 1 . This agreement, particularly for the case of small and moderate values of $q$, unambiguously evidences that Eq. (71) can be used in a wide space of parameters for the characterization of diverse modes in both natural and artificial biaxial crystal slabs.

\section{CONCLUSIONS}

To summarize, we have presented an analytical derivation of the electromagnetic modes that can be guided along biaxial crystal slabs. We have provided simple expressions for the dispersion of the modes in the limit of an ultrathin slab and for the case of large $k$-vectors of the modes. Both limits are currently of great importance for studying highly confined anisotropic polaritons in vdW biaxial crystal slabs and, particularly, for the interpretation of the state-of-the-art near-field experiments. 


\section{ACKNOWLEDGMENTS}

We thank A. Bylinkin for checking the analytical derivations. A.Y.N. acknowledges the Spanish Ministry of Science, Innovation and Universities (national Project No. MAT2017-88358-C3-3-R) and Basque Government
(Grant No. IT1164-19). P.A.-G. acknowledges support from the European Research Council under Starting Grant No. 715496, 2DNANOPTICA. K.V.V. and V.S.V. acknowledge support from the Russian Science Foundation, Grant No. 18-19-00684.

G.Á-P. and K.V.V. contributed equally to this work.
[1] D. S. Kliger, Polarized Light in Optics and Spectroscopy (Academic, New York, 1990).

[2] E. Yablonovitch, Phys. Rev. Lett. 58, 2059 (1987).

[3] W. L. Barnes, A. Dereux, and T. W. Ebbesen, Nature (London) 424, 824 (2003).

[4] J. B. Pendry, L. Martín-Moreno, and F. J. García-Vidal, Science 305, 847 (2004).

[5] N. Yu and F. Capasso, Nat. Mater. 13, 139 (2014).

[6] A. V. Kildishev, A. Boltasseva, and V. M. Shalaev, Science 339, 1232009 (2013).

[7] E. Cubukcu, K. Aydin, E. Ozbay, S. Foteinopoulou, and C. M. Soukoulis, Nature (London) 423, 604 (2003).

[8] T. Baba, Nat. Photonics 2, 465 (2008).

[9] J. B. Pendry, Phys. Rev. Lett. 85, 3966 (2000).

[10] T. Taubner, D. Korobkin, Y. Urzhumov, G. Shvets, and R. Hillenbrand, Science 313, 1595 (2006).

[11] A. K. Geim and I. V. Grigorieva, Nature (London) 499, 419 (2013).

[12] D. N. Basov, M. M. Fogler, and F. J. G. de Abajo, Science 354, 195 (2016).

[13] T. Low, A. Chaves, J. D. Caldwell, A. Kumar, N. X. Fang, P. Avouris, T. F. Heinz, F. Guinea, L. Martín-Moreno, and F. Koppens, Nat. Mater. 16, 182 (2017).

[14] S. Dai, Z. Fei, Q. Ma, A. S. Rodin, M. Wagner, A. S. McLeod, M. K. Liu, W. Gannett, W. Regan, K. Watanabe, T. Taniguchi, M. Thiemens, G. Dominguez, A. H. C. Neto, A. Zettl, F. Keilmann, P. Jarillo-Herrero, M. M. Fogler, and D. N. Basov, Science 343, 1125 (2014).

[15] P. Li, I. Dolado, F. J. Alfaro-Mozaz, F. Casanova, L. E. Hueso, S. Liu, J. H. Edgar, A. Y. Nikitin, S. Vélez, and R. Hillenbrand, Science 359, 892 (2018).

[16] W. Ma, P. Alonso-González, S. Li, A. Y. Nikitin, J. Yuan, J. Martín-Sánchez, J. Taboada-Gutiérrez, I. Amenabar, P. Li, S. Vélez, C. Tollan, Z. Dai, Y. Zhang, S. Sriram, K. KalantarZadeh, S.-T. Lee, R. Hillenbrand, and Q. Bao, Nature (London) 562, 557 (2018).

[17] S. Dai, Q. Ma, T. Andersen, A. S. Mcleod, Z. Fei, M. K. Liu, M. Wagner, K. Watanabe, T. Taniguchi, M. Thiemens,
F. Keilmann, P. Jarillo-Herrero, M. M. Fogler, and D. N. Basov, Nat. Commun. 6, 6963 (2015).

[18] Z. Zheng, N. Xu, S. L. Oscurato, M. Tamagnone, F. Sun, Y. Jiang, Y. Ke, J. Chen, W. Huang, W. L. Wilson, A. Ambrosio, S. Deng, and H. Chen, Sci. Adv. 5, eaav8690 (2018).

[19] M. I. D’yakonov, Zh. Eksp. Teor. Fiz. 94, 119 (1988) [Sov. Phys. JETP 67, 714 (1988)].

[20] O. Takayama, L.-C. Crasovan, S. K. Johansen, D. Mihalache, D. Artigas, and L. Torner, Electromagnetics 28, 126 (2008).

[21] E. E. Narimanov, Phys. Rev. A 98, 013818 (2018).

[22] T. Maldonado and T. Gaylord, J. Lightwave Technol. 14, 486 (1996).

[23] M. S. Kharusi, J. Opt. Soc. Am. 64, 27 (1974).

[24] N. Jovanovic and W. Papousek, Int. J. Electron. Commun. 55, 123 (2001).

[25] Z. Chen and Z. Shen, Appl. Sci. 8, 102 (2018).

[26] L. D. Landáu and Y. M. Lífshits, Electrodynamics of Continuous Media, Vol. 8 (Pergamon, Oxford, 1960), p. 324.

[27] M. Born, E. Wolf, A. B. Bhatia, P. C. Clemmow, D. Gabor, A. R. Stokes, A. M. Taylor, P. A. Wayman, and W. L. Wilcock, Principles of Optics: Electromagnetic Theory of Propagation, Interference and Diffraction of Light, 7th ed. (Cambridge University Press, Cambridge, 1999).

[28] V. Agranovich and D. Mills, Surface Polaritons: Electromagnetic Waves at Surfaces and Interfaces (Elsevier, Amsterdam, 1982), p. 35.

[29] P. Gonçalves and N. Peres, An Introduction to Graphene Plasmonics (World Scientific, Singapore, 2016), p. 464.

[30] J. D. Caldwell, I. Aharonovich, G. Cassabois, J. H. Edgar, B. Gil, and D. N. Basov, Nat. Rev. Mater. 4, 552 (2019).

[31] A. Y. Nikitin, Graphene Plasmonics. World Scientific Handbook of Metamaterials and Plasmonics. World Scientific Series in Nanoscience and Nanotechnology, Vol. 4 (Cambridge University Press, Cambridge, 2017), Chap. 8, pp. 307-338.

[32] J. S. Gómez-Díaz, M. Tymchenko, and A. Alù, Phys. Rev. Lett. 114, 233901 (2015).

[33] O. Y. Yermakov, A. I. Ovcharenko, M. Song, A. A. Bogdanov, I. V. Iorsh, and Y. S. Kivshar, Phys. Rev. B 91, 235423 (2015).

[34] G. W. Hanson, J. Appl. Phys. 103, 064302 (2008). 